\title{
Open resource metagenomics: a model for sharing metagenomic libraries
}

\author{
J.D. Neufeld ${ }^{1}$, K. Engel' ${ }^{1}$ J. Cheng ${ }^{1}$, G. Moreno-Hagelsieb ${ }^{2}$, D.R. Rose', T.C. Charles ${ }^{1}$ \\ ${ }^{1}$ University of Waterloo, Department of Biology, Waterloo, ON, Canada \\ ${ }^{2}$ Wilfrid Laurier University, Department of Biology, Waterloo, ON, Canada
}

Corresponding author: tcharles@uwaterloo.ca

Keywords: sharing, metagenomic libraries, functional complementation

\begin{abstract}
Both sequence-based and activity-based exploitation of environmental DNA have provided unprecedented access to the genomic content of cultivated and uncultivated microorganisms. Although researchers deposit microbial strains in culture collections and DNA sequences in databases, activity-based metagenomic studies typically only publish sequences from the hits retrieved from specific screens. Physical metagenomic libraries, conceptually similar to entire sequence datasets, are usually not straightforward to obtain by interested parties subsequent to publication. In order to facilitate unrestricted distribution of metagenomic libraries, we propose the adoption of open resource metagenomics, in line with the trend towards open access publishing, and similar to culture- and mutant-strain collections that have been the backbone of traditional microbiology and microbial genetics. The concept of open resource metagenomics includes preparation of physical DNA libraries, preferably in versatile vectors that facilitate screening in a diversity of host organisms, and pooling of clones so that single aliquots containing complete libraries can be easily distributed upon request. Database deposition of associated metadata and sequence data for each library provides researchers with information to select the most appropriate libraries for further research projects. As a starting point, we have established the Canadian MetaMicroBiome Library (CM ${ }^{2} \mathrm{BL}$ [1]). The $\mathrm{CM}^{2} \mathrm{BL}$ is a publicly accessible collection of cosmid libraries containing environmental DNA from soils collected from across Canada, spanning multiple biomes. The libraries were constructed such that the cloned DNA can be easily transferred to Gateway® compliant vectors, facilitating functional screening in virtually any surrogate microbial host for which there are available plasmid vectors. The libraries, which we are placing in the public domain, will be distributed upon request without restriction to members of both the academic research community and industry. This article invites the scientific community to adopt this philosophy of open resource metagenomics to extend the utility of functional metagenomics beyond initial publication, circumventing the need to start from scratch with each new research project.
\end{abstract}

\section{Introduction}

Microbial communities harbor the immense genetic diversity that controls Earth's biogeochemical cycling. This genetic diversity has a concomitantly immense potential for applications in bio-product synthesis, green chemistry and pharmaceutical and bio-energy sectors. Metagenomic libraries provide a window into this largely untapped reservoir of nucleic acid diversity. Individual metagenomic libraries have been generated from a variety of terrestrial and aquatic environments, and these have been prepared either as sequence-based libraries for analysis and submission to public databases, or as DNA libraries captured in a host organism (usually Escherichia coli) and stored as a collection of clones.

Sequencing technologies have progressed over the past decade to the point where the size of new sequence-based metagenomic and metatranscriptomic datasets often eclipses the sum of all previous database collections. Although the complete assembly of genomes from DNA sequence data generated from most environmental samples is still usually foiled by the immense genetic diversity in most microbial communities, the annotation and comparison of metagenomic sequence data can reveal functional trends that 
help explain adaptations of microbes to their respective habitats. Environmental sampling and sequence collection have been made possible in even modestly funded research laboratories by the advent of post-Sanger sequence platforms. Given the deluge of sequence data, the Genomic Standards Consortium (GSC) has recognized the drawbacks of the lack of sample metadata submission, or the submission of metadata structured at the discretion of individual researchers [2,3]; the recent publication of minimum information about any $(\mathrm{x})$ sequence (MIXS) specifications represents a current example of essential metadata standards for adoption by the scientific community [4]. These standards will provide order and consistency as DNA sequence analysis of microbial communities continues to expand.

While the scientific community recognizes the importance of appropriate metadata collection for genomics and metagenomics research, the accompanying physical metagenomic libraries are not commonly generated and maintained as shared resources. Since the publication of the first metagenomic libraries from marine water samples [5,6], nucleic acids from diverse terrestrial, aquatic and host-associated environments have been captured in plasmid, fosmid, cosmid or bacterial artificial chromosome (BAC) libraries. The construction of these libraries requires considerable effort on the part of highly skilled bench scientists. The ends of these cloned fragments are often sequenced but, importantly, the libraries themselves are subjected to functional screening or selection for specific genes and functions. However, nearly every functional metagenomics study follows a similar methodological approach: researcher collects samples, constructs a metagenomic library, retrieves clones of immediate interest for further analysis, and stores the library until publication or a later time point. For each new study, the collection and repetition of each step could be rendered unnecessary if appropriate previously constructed libraries were available.

\section{Physical metagenomic libraries}

Given that the success of a phenotypic screen or selection hinges on the screening strategy, expression host and the function being sought, the libraries are able to yield nearly limitless value for additional combinations and comparisons in later studies. Several examples already exist of individual laboratories mining the same metagenomic libraries for myriad functions across multiple studies. One of the earliest metagenomic studies involved capturing soil DNA in large-insert BAC libraries, followed by screens to identify clones that coded for activities including DNase, antibacterial, lipase, amylase, cellulase, chitinase, esterase, keratinase and protease [7]. The same soil library (SL2) was then used for (a) screening and recovery of genes coding for the production of turbomycin A and B [8], (b) linking ribosomal RNA genes with additional genetic material [9] and (c) identifying clones conferring antibiotic resistance [10]. All of these SL2 screens were done in E. coli as the host. Another example of multiple applications for metagenomic libraries includes cosmid libraries generated from activated sludge and soils. These originally underwent selection for clones conferring the ability to utilize D3-hydroxybutyrate as sole carbon source in both $E$. coli and Ensifer meliloti (Sinorhizobium meliloti) surrogate hosts [11]. Subsequently, several of these libraries were screened to retrieve luxR-luxI type quorum sensing systems [12], phosphate metabolism genes [13], and poly-3-hydroxybutyrate synthesis genes [14], all in E. meliloti or Agrobacterium tumefaciens as a surrogate host. These examples illustrate the sustained value of metagenomic libraries for use in a range of hosts for uncovering a wide variety of different genes, enzymes and functions related to small-molecule metabolites in the environment.

\section{Sharing model in science}

Unlike the examples above, most metagenomic libraries are prepared for individual projects and are not necessarily maintained in a manner that facilitates convenient and low-cost distribution. This situation hinders both repetition of the work done previously and exploration of the captured DNA for additional enzymatic functions. These libraries, similar to cultured isolates or mutant strains, could have value extending far beyond the initial publication. According to the International Journal of Systematic and Evolutionary Microbiology, "characterization of prokaryote strains must include all relevant metadata (e.g. location isolated, strain designations and culture collections, and other environmental variables)" [15]. For mutant strains, the Molecular Microbiology journal stipulates that "authors will distribute freely any strains, clones or antibodies described therein for use in academic research", which is similar to the recommendation for authors from the Journal of Bacteriology that mutant strains 
be "available from a national collection or will be made available in a timely fashion, at reasonable cost, and in limited quantities to members of the scientific community for noncommercial purposes".

A tradition of sharing has been integral to the development of the science of microbiology. From the earliest days, sharing of pure cultures has been essential to experimental replication and validation, and for the definition of bacterial types. The validity of patent claims requires access to cultures, and such access is legally mandated. Central culture collections such as the American Type Culture Collection (ATCC), Deutsche Sammlung von Mikroorganismen und Zellkulturen (DSMZ) and hundreds of others around the world [16] have ensured strain availability and the scientific community has rallied to ensure strain maintenance [17]. These conventions do not yet have equivalents for metagenomic libraries.

One reason for the lack of storage and distribution standards for physical metagenomic libraries may be that the customary formats for storage are 96or 384-well plates. Multi-well plates require extensive storage space. For example, a single 100,000-clone metagenomic library from a soil sample would be represented by approximately $\sim 1000$ 96-well plates or $\sim 250$ 384-well plates, which could fill an entire ultrafreezer with only 96-well plates. Due to the obvious challenges of permanent storage of large numbers of platearrayed metagenomic libraries, we argue that libraries should also be preserved in a format that is amenable to low-cost shipping and storage so these valuable resources remain available for long-term distribution subsequent to publication.

\section{Open resource metagenomics}

We propose an open resource model for archiving published libraries using an alternative clone pooling strategy for storage and distribution. The features of this model include (a) cloning of largesize inserts into versatile vectors for downstream screening or selection in a variety of hosts, (b) pooling of clones for facilitating convenient library distribution in standard microcentrifuge tubes, and (c) extensive metadata and associated sequence-based characterization of libraries.

Cloning of inserts into broad host-range vectors helps circumvent one of the main limitations of metagenomic library functional screens: the inability to express many genes in a single host (e.g. E. coli). Indeed, screening in multiple hosts can reveal target clones that would not have been evident otherwise [11]. Because successful expression of heterologous genes is dependent on the surrogate host employed, it is desirable to construct libraries in vectors compatible with a variety of host organisms. Given the specific hostrange requirements of different library vectors, it is unlikely that a universal vector, able to replicate in all possible hosts, will be developed. Instead, one approach is to employ Gateway ${ }^{\circledR}$ technology [18] in cosmid library vectors to enable en masse transfer of cloned genomic DNA from the library vector backbone to host-specific destination vectors, resulting in expression clones appropriate for the desired surrogate host.

Our recommended protocol for pooling clones is less labor intensive than picking colonies for distribution into multi-well plates. Instead of growth and storage of arrays of clones in individual wells, we suggest that libraries initially grown as colonies be sequentially washed from agar surfaces with fresh liquid culture medium. Alternatively, selection of clones during library construction can be carried out directly in liquid culture as demonstrated previously [19]. The resulting volume of medium with a dense collection of clones can be distributed into frozen aliquots for future dilution and plating for screens, or transfer to defined media for targeted selection protocols. These colony aliquots or DNA preparations can be shipped to collaborating laboratories for further investigation (e.g. the SL2 library has been distributed in this way; Jo Handelsman, personal communication).

To facilitate direct access to these libraries by members of the worldwide scientific community, sample information can be retrieved from a selection of database fields such as the environmental context, the preparation method, and a link to a sequence annotation server such as MGRAST [20] where associated sequence data, and sequence-based phylogenetic and metabolic information can be hosted. The cost of sequencing has decreased such that bulk DNA and 16S rRNA gene sequences can be obtained affordably to provide large-scale phylogenetic and metabolic characterization of each environmental sample. These sequence data represent an important complement to detailed physical, chemical and geographic information that comprise the MIxS specifications. Internally, the Handlebar database [21] can allocate unique barcodes for samples and libraries. 
Altogether, these approaches are affordable and would result in libraries being made readily available to all researchers, and allow future grant proposals to leverage existing libraries from international research colleagues. Each functional study using the library could be related to the sequence data, as well as other functional studies using the same library, and would ensure that the original creators of the library are appropriately cited. Open resource metagenomics helps circumvent the traditional cycle of requiring new samples to be collected for each subsequent study. With such an approach, metagenomic libraries could be considered akin to isolates and mutant strains that are housed in culture collections. Unlike established culture collections, our recommendation would be that open resource metagenomics be established conceptually as a federated system in which individual laboratories maintain control and maintenance of pooled metagenomic libraries, ready for distribution upon request. As the ethos of open resource metagenomics and demand for access to libraries builds, it is not inconceivable that central repositories (e.g. ATCC, DSMZ) could be approached to offer permanent storage for these genetic resources. Ultimately, the scientific value of environmental metagenomic libraries is analogous to the agricultural value of seeds from Earth's plants, which are protected within a distributed system of Seedbanks.

Of course, as with isolates and mutant strains, there remain outstanding challenges that could prevent universal adoption of open resource metagenomics. For example, many funding agencies require and encourage the involvement of for-profit industry, which typically includes agreements with regards to intellectual property. Under these circumstances, the reality may be that unique arrangements will be required to blend open resource metagenomics with industrial collaborations. These arrangements may include delayed library release or the maintenance of particular libraries outside of the research literature. In addition, some laboratories may require a specific security level for labs handling and receiving metagenomic material and others may require signed agreements for transfers of research materials. Of course another important barrier to a central repository would be financial implications. As with culture collections, sustained funding is essential to maintain resources for sample submission, cataloguing and distribution. Although we are seeking appropriate funding towards establishing such a centralized system, the methodological approaches outlined above will enable the immediate adoption of a distributed open resource system for researchers preparing new metagenomic libraries. These are issues that we are working to resolve and we urge the research community to join this effort in moving toward a system of open resource metagenomics for greatest international collaborative benefit.

\section{Starting point initiative}

In order to demonstrate that such an open resource approach is feasible, we have initiated the Canadian MetaMicroBiome Library project ( $\mathrm{CM}^{2} \mathrm{BL}$ [1]), a publicly accessible collection of libraries of environmental DNA representing Canadian soil microbial communities. Canada spans a variety of natural regions, or ecozones (Figure 1). Its 20 ecozones consist of 15 terrestrial and 5 marine regions. The $\mathrm{CM}^{2} \mathrm{BL}$ was initiated with the construction of cosmid libraries containing DNA isolated from soil samples collected from across Canada spanning multiple biomes and ecozones (Figure 1). These samples are being characterized by high-throughput DNA sequencing to determine the taxonomic, genetic, and metabolic diversity of each sample alongside screens for industrially relevant enzymes. Once the proof-of-principle stage of $\mathrm{CM}^{2} \mathrm{BL}$ is complete, contributions of samples from other environments will be considered for inclusion, provided additional samples are sufficiently distinct to maximize captured microbial diversity. The resulting resources will provide the international scientific community with access to a large collection of thoroughly characterized genetic materials in clone library format for phenotypic screening or selection for enzymes with truly novel functions, independent from sequence-based surveys. We invite other scientists who are regularly generating libraries to make them publicly available in a similar manner, and to work with us to develop an exchange system so that libraries are mirrored in multiple archive locations. 


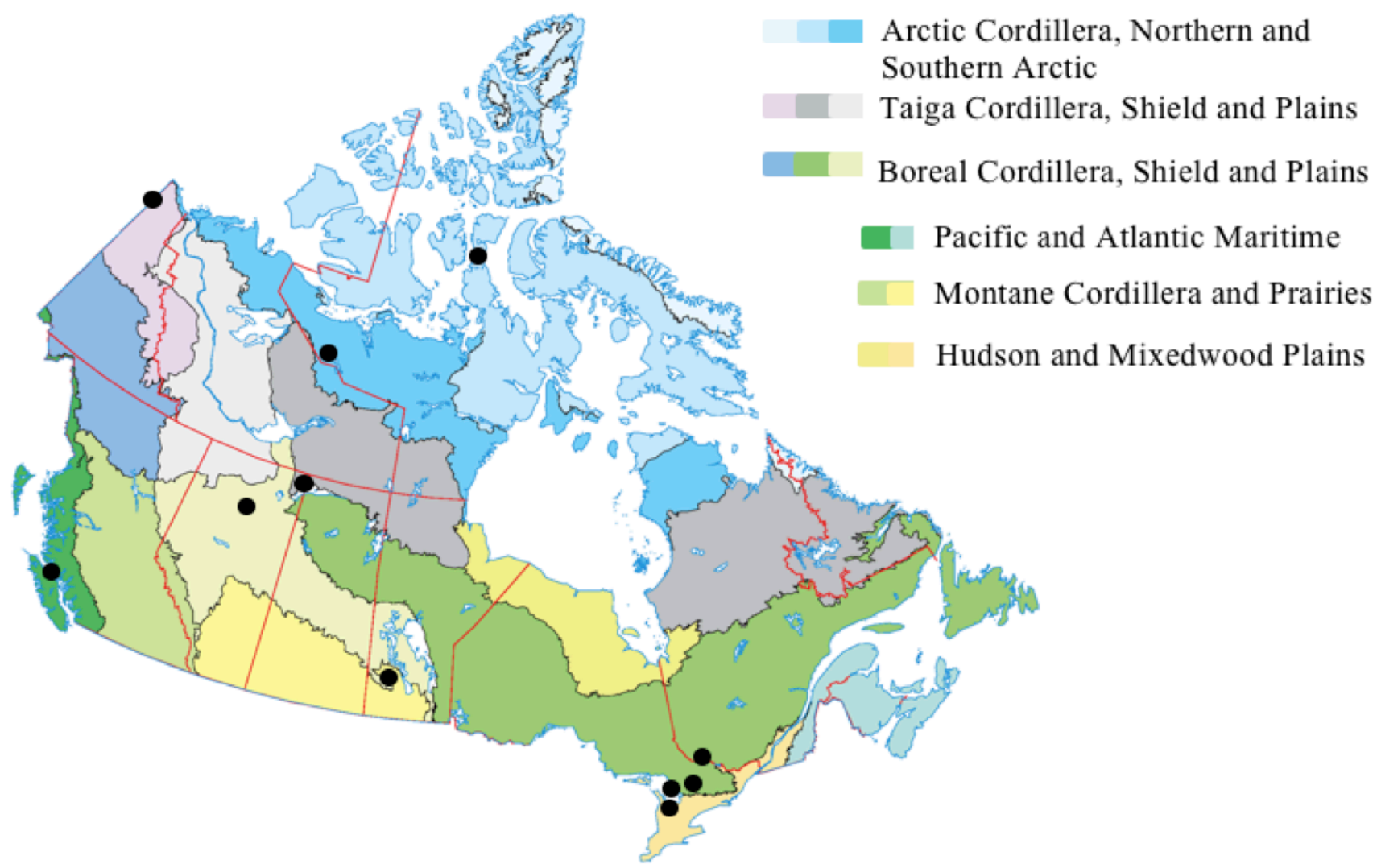

Figure 1. Terrestrial ecozones of Canada. The location of initial soil samples for the Canadian MetaMicroBiome Library is indicated with a black circle $(\bullet)$. The map was modified from the Canadian Soil Information System.

The $\mathrm{CM}^{2} \mathrm{BL}$ libraries are prepared using derivatives of the IncP cosmid pRK7813 [22] that have been converted to Gateway® entry vectors. This allows the insert DNA to be easily transferred to Gateway® vectors of diverse host range using either in vivo [23] or in vitro [24] reactions, thus facilitating phenotypic screening of the libraries in bacterial and yeast hosts of interest. We store backups at various stages of library development: DNA extracts, ligation products, packaged phage particles, clone libraries in E. coli and as extracted cosmid clone DNA. For each sample prepared thus far, libraries contain pools of between 10,000 and 250,000 cosmids. Copies of these libraries are stored as permanent frozen archives and purified cosmid DNA, in duplicate freezers with $\mathrm{CO}_{2}$ backup systems to ensure security of the resource. If required, additional library expansions can be generated for distribution to companies or academic institutions at minimal cost per library.
Distribution of libraries is accompanied by materials transfer agreements to ensure consistency with requirements of the UN Convention on Biodiversity [25].

The $\mathrm{CM}^{2} \mathrm{BL}$ resource was initiated by preparing a cornfield soil metagenomic library using the versatile IncP cosmid vector pJC8 (pRK7813 derivative; Figure 2), allowing for phenotypic screening in a broad range of microbial surrogate hosts. Cosmid pJC8 is a low-copy and broad-hostrange cosmid. The cosmid library was constructed using Stratagene packaging extracts and E. coli HB101 (3.2 × $10^{5}$ clones formed/ $\mu$ g insert DNA). A total of 79,058 clones with $\sim 33 \mathrm{~kb}$ random inserts have been generated. The resulting soil DNA library was calculated to contain $2,640 \mathrm{Mb}$ of metagenomic DNA, which represents approximately 561 genomes, assuming average bacterial genome size of $4.7 \mathrm{Mb}$ in the soil sample [26]. 


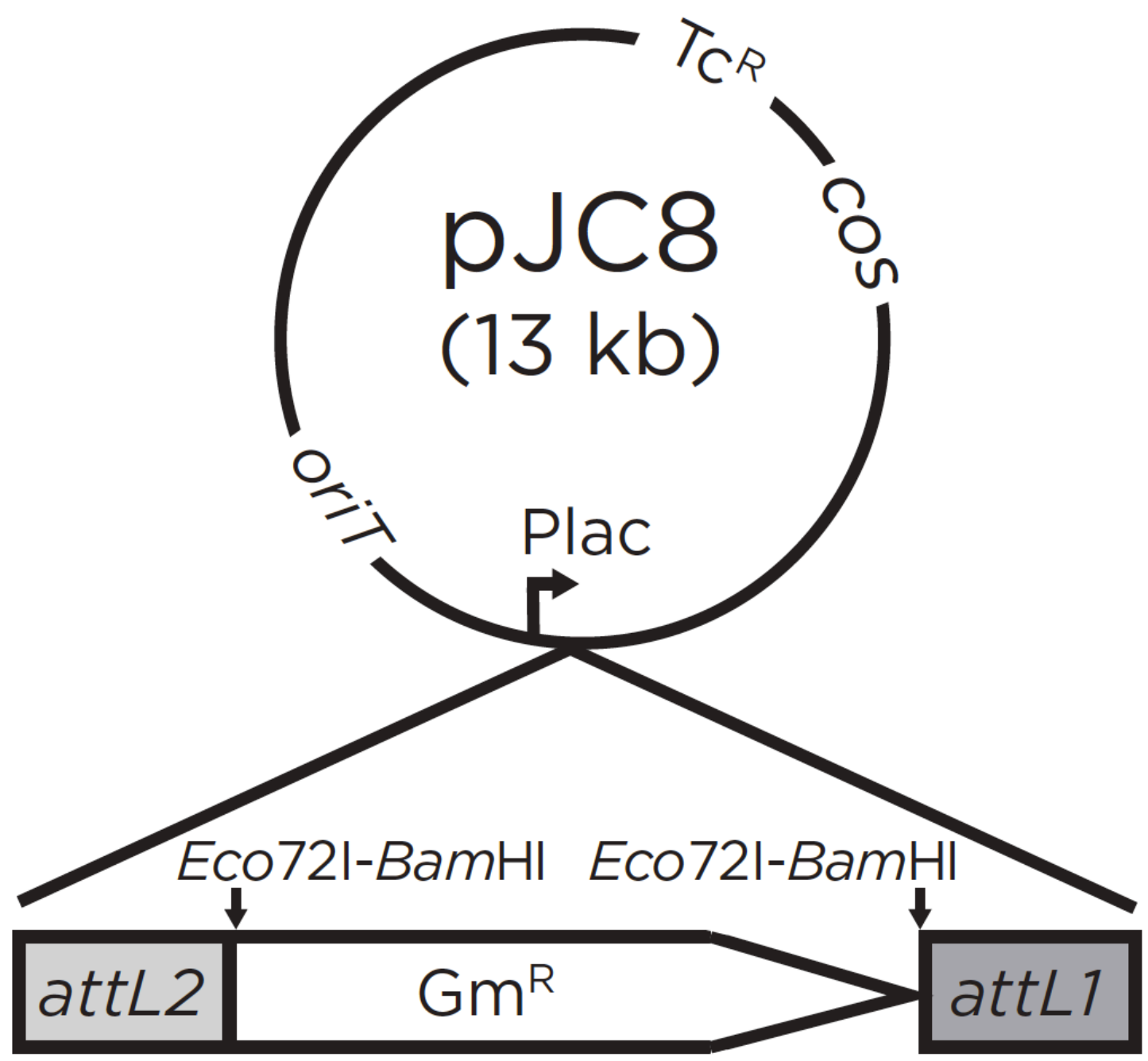

Figure 2. Gateway ${ }^{\circledR}$ entry cosmid pJC8. BamHI and $E_{C O} 72 \mathrm{I}$ sites are used for cloning sticky and blunt ends of inserts, respectively. The cos site is used for in vitro packaging of the recombinant cosmid DNA into bacteriophage $\lambda$ heads; oriT (RK2 origin of transfer) site is used for the transfer of cosmid clones from $E$. coli to other bacterial hosts.

\section{Conclusion}

Considering that methods for preparing metagenomic libraries have largely remained constant since their conception in the 1990s, these proposed standards for metadata, storage and distribution of metagenomic libraries are anticipated to remain relevant for decades. We are expanding $\mathrm{CM}^{2} \mathrm{BL}$ to include samples from additional environments and have partnered with international research initiatives such as the Earth Microbiome Project to provide sequence-based

\section{Acknowledgments}

We thank Jack Gilbert, Mark Liles, Angela Sessitsch and Wolfgang Streit for their comments and suggestions in context for libraries intended for functional screens and selections. Ultimately, open resource metagenomics, and initiatives such as $\mathrm{CM}^{2} \mathrm{BL}$, will provide the international scientific community with cost-recovery access to a collection of thoroughly characterized genetic materials for the phenotypic screening of enzymes and other gene products with truly novel functions independent of sequence-based surveys, available for distribution upon request.

the preparation of this manuscript. This work was supported by an NSERC Strategic Projects grant. 


\section{References}

1. Canadian MetaMicroBiome Library. http://cm2bl.org

2. Garrity GM, Field D, Kyrpides N, Hirschman L, Sansone SA, Angiuoli S, Cole JR, Glöckner FO, Kolker E, Kowalchuk G, et al. Toward a standards-compliant genomic and metagenomic publication record. OMICS 2008; 12:157-160. PubMed doi:10.1089/omi.2008.A2B2

3. Field D, Garrity G, Gray T, Morrison N, Selengut J, Sterk P, Tatusova T, Thomson N, Allen MJ, Angiuoli SV, et al. The minimum information about a genome sequence (MIGS) specification. Nat Biotechnol 2008; 26:541-547. PubMed doi:10.1038/nbt1360

4. Yilmaz P, Kottmann R, Field D, Knight R, Cole JR, Amaral-Zettler L, Gilbert JA, Karsch-Mizrachi I, Johnston A, Cochrane G, et al. Minimum information about a marker gene sequence (MIMARKS) and minimum information about any (x) sequence (MIxS) specifications. Nat Biotechnol 2011; 29:415-420. PubMed doi:10.1038/nbt.1823

5. Stein JL, Marsh TL, Wu KY, Shizuya H, DeLong EF. Characterization of uncultivated prokaryotes: isolation and analysis of a 40-kilobase-pair genome fragment from a planktonic marine archaeon. J Bacteriol 1996; 178:591-599. PubMed

6. Schmidt TM, DeLong EF, Pace NR. Analysis of a marine picoplankton community by $16 \mathrm{~S}$ rRNA gene cloning and sequencing. I Bacteriol 1991; 173:4371-4378. PubMed

7. Rondon MR, August PR, Bettermann AD, Brady SF, Grossman TH, Liles MR, Loiacono KA, Lynch BA, MacNeil IA, Minor C, et al. Cloning the soil metagenome: a strategy for accessing the genetic and functional diversity of uncultured microorganisms. Appl Environ Microbiol 2000;

66:2541-2547. PubMed doi:10.1128/AEM.66.6.2541-2547.2000

8. Gillespie DE, Brady SF, Bettermann AD, Cianciotto NP, Liles MR, Rondon MR, Clardy J, Goodman RM, Handelsman J. Isolation of antibiotics turbomycin $\mathrm{a}$ and $\mathrm{B}$ from $\mathrm{a}$ metagenomic library of soil microbial DNA. Appl Environ Microbiol 2002; 68:4301-4306. PubMed doi:10.1128/AEM.68.9.4301-4306.2002

9. Liles MR, Manske BF, Bintrim SB, Handelsman J, Goodman RM. A census of rRNA genes and linked genomic sequences within a soil metagenomic library. App/ Environ Microbiol
2003; 69:2684-2691. PubMed

doi:10.1128/AEM.69.5.2684-2691.2003

10. Riesenfeld CS, Goodman RM, Handelsman J. Uncultured soil bacteria are a reservoir of new antibiotic resistance genes. Environ Microbiol 2004; 6:981-989. PubMed doi:10.1111/j.14622920.2004.00664.x

11. Wang C, Meek DJ, Panchal P, Boruvka N, Archibald FS, Driscoll BT, Charles TC. Isolation of poly-3-hydroxybutyrate metabolism genes from complex microbial communities by phenotypic complementation of bacterial mutants. Appl Environ Microbiol 2006; 72:384-391. PubMed doi:10.1128/AEM.72.1.384-391.2006

12. Hao Y, Winans SC, Glick BR, Charles TC. Identification and characterization of new LuxR/Luxl-type quorum sensing systems from metagenomic libraries. Environ Microbiol 2010; 12:105-117. PubMed doi:10.1111/j.14622920.2009.02049.x

13. Rolider A. Isolation and characterization of bacterial phosphorous metabolism genes from complex microbial communities. 2009; PhD thesis, University of Waterloo, http://hdl.handle.net/10012/4806

14. Schallmey M, Ly A, Wang C, Meglei G, Voget S, Streit WR, Driscoll BT, Charles TC. Harvesting of novel polyhydroxyalkanoate (PHA) synthase encoding genes from soil metagenome libraries using phenotypic screening and selection. FEMS Microbiol Lett 2011; 321:150-156. PubMed doi:10.1111/j.1574-6968.2011.02324.x

15. Tindall BJ, Rossello-Mora R, Busse HJ, Ludwig W, Kampfer P. Notes on the characterization of prokaryote strains for taxonomic purposes. Int J Syst Evol Microbiol 2010; 60:249-266. PubMed doi:10.1099/ijs.0.016949-0

16. World Federation for Culture Collections. http://www.wfcc.info

17. Ward N, Eisen J, Fraser C, Stackebrandt E. Sequenced strains must be saved from extinction. Nature 2001; 414:148. PubMed doi: $10.1038 / 35102737$

18. Katzen F. Gateway $®$ recombinational cloning: a biological operating system. Expert Opin Drug Discov 2007; 2:571-589. doi:10.1517/17460441.2.4.571

19. Kim JH, Feng Z, Bauer JD, Kallifidas D, Calle PY, Brady SF. Cloning large natural product gene clusters from the environment: Piecing 
environmental DNA gene clusters back together with TAR. Biopolymers 2010; 93:833-844. PubMed doi:10.1002/bip.21450

20. Meyer F, Paarmann D, D'Souza M, Olson R, Glass E, Kubal M, Paczian T, Rodriguez A, Stevens R, Wilke A. The metagenomics RAST server - a public resource for the automatic phylogenetic and functional analysis of metagenomes. BMC Bioinformatics 2008; 9:386. PubMed doi:10.1186/1471-2105-9-386

21. Booth T, Gilbert J, Neufeld J, Ball J, Thurston M, Chipman K, Joint I, Field D. Handlebar: a flexible, web-based inventory manager for handling barcoded samples. Biotechniques 2007; 42:300302. PubMed doi:10.2144/000112385

22. Jones JD, Gutterson N. An efficient mobilizable cosmid vector, pRK7813, and its use in a rapid method for marker exchange in Pseudomonas fluorescens strain HV37a. Gene 1987; 61:299306. PubMed doi:10.1016/0378-1119(87)90193$\underline{4}$
23. House BL, Mortimer MW, Kahn ML. New recombination methods for Sinorhizobium meliloti genetics. Appl Environ Microbiol 2004; 70:2806-2815. PubMed doi:10.1128/AEM.70.5.2806-2815.2004

24. Hartley JL, Temple GF, Brasch MA. DNA cloning using in vitro site-specific recombination. Genome Res 2000; 10:1788-1795. PubMed doi:10.1101/gr.143000

25. Garrity GM, Thompson LM, Ussery DW, Paskin $\mathrm{N}$, Baker D, Desmeth P, Schindel DE, Ong PS. Studies on monitoring and tracking genetic resources: an executive summary. Stand Genomic Sci 2009; 1:78-86. PubMed doi:10.4056/sigs.1491

26. Raes J, Korbel J, Lercher M, Von Mering C, Bork P. Prediction of effective genome size in metagenomic samples. Genome Biol 2007; 8:R10. PubMed doi:10.1186/gb-2007-8-1-r10 